\title{
Efficiency Evaluation Model of Students' Outdoor Taichi Training Based on Supervised Learning
}

\author{
Wang Youjun \\ School of Taichi and Martial Arts, Jiaozuo University, Jiaozuo, Henan, China
}

\begin{abstract}
:
Taichi has a long history and spread widely in China. It has played an important role in maintaining human health. This paper studies the efficiency evaluation model of students' outdoor Taichi training based on supervised learning. This paper expounds the key points and importance of sports health preservation through the collation of sports health preservation related literature, the comparison between traditional sports methods and modern sports methods, and the interpretation of knowledge and practice Taichi. This paper analyzes the influence of Taichi on the total score of body symptoms. Based on supervised learning algorithm, this paper compares Taichi with modern sports and irregular sports, studies the effect of Taichi on body conditioning, and puts forward an evaluation model of Taichi training efficiency. The results obtained in this paper lay a theoretical foundation for the promotion of knowledge and practice Taichi, and provide data reference for the establishment of Taichi training efficiency.
\end{abstract}

Keywords: Taichi, Supervised Learning, Outdoor Sports, Training Efficiency Evaluation Model.

\section{INTRODUCTION}

In order to make it convenient for people, especially young and middle-aged people, to relieve their tired bodies from their busy schedule and take some time to move easily and reduce pressure, a set of eight style knowledge and practice Taichi has been created, which takes only one minute at the fastest [1-2]. The simple eight moves of knowing and doing Tai Chi are easy to absorb and master. They are very simple to understand. They are cognitive behavioral therapy that can deal with personal body, mouth and mind. They are designed to enable people to recognize the coordination of physical condition and psychological thinking, the balance concept of body, mouth and mind and standardized exercise [3]. They can cultivate both inside and outside to achieve the effect of physical and mental co cultivation. 
Article History: Received: 28 October 2021 Revised: 05 December 2021 Accepted: 10 January 2022 Publication: 28 February 2022

Can the simplified knowledge and practice Taichi still achieve good physical and mental recuperation? This topic aims to classify and analyze the theory of fitness effect of Zhixing Taichi through literature research, explore the difference between traditional sports health preservation methods and modern sports methods, and sort out and study the theory and origin of Zhixing Taichi. By using SCL-90 symptom checklist, the changes of total symptom scores before and after the investigation were compared among the knowledge and practice Taichi group, modern exercise group and blank control group [4-5]. By comparison and analysis, this paper studies the efficiency of knowledge and practice Taichi in physical and mental conditioning effect, so as to provide theoretical and practical basis for the promotion of simple and easy knowledge and practice Taichi, so as to enrich the content of sports health preservation and carry forward Chinese traditional health preservation culture!

\section{INTRODUCTION TO ZHIXING Taichi}

\section{A philosophical study of knowing and doing Taichi}

Tai Chi is a philosophical term in ancient China, which was first seen in the book of changes: "Yi has Tai Chi, which produces Liangyi, Liangyi produces Sixiang, Sixiang produces Bagua, Bagua determines good or bad luck, and good or bad luck produces great cause" [6]. "Too" means the greatest; "Ji" has the meaning of limit. Tai Chi is the origin of limit and derived everything. The book of changes also reveals the way of Yin-Yang change of Tai Chi, which means "poverty leads to change, change leads to flexibility, which means change also" [7]. The Tai Chi diagram is the best interpretation of the principle of Tai Chi. The yin-yang half arc pattern vividly summarizes the rules, principles, mysteries and modifications of all things in the universe, and shows the fundamental principle of the growth of all things - opposites and complements each other. Taichi, named after Taichi, symbolizes that Taichi is circular and circulating. Its boxing theory, boxing and Taoism are natural, including heaven and earth, light and flexible, and the combination of hardness and softness [8].

The word "knowing and doing" first appeared in the book of history. "It is not difficult to know, but difficult to do", which means "it is easy to know but difficult to do". This view was more common in the spring and Autumn period. In the 12th year of Duke Xuangong (597 BC), Zuo Zhuan recorded: "advance when you can, retreat when you know difficulties, and good governance in the army." in the first year of Duke Chenggong (590 BC), Zuo Zhuan recorded: "you can succeed if you are prepared when you know difficulties." (Zuo Zhuan recorded in the third year of Duke Zhaogong (539 BC): "the gentleman said: it is difficult to know, but it is impossible to follow when you know them." (Zuo Zhuan recorded in the tenth year of Duke Zhaogong (532 BC): "If you don't know the truth, you will be good at it."

It can be seen that in the spring and Autumn period, "knowing and doing" has become an issue of concern. The first two say that "knowing first and then later", knowing difficulties can 
Article History: Received: 28 October 2021 Revised: 05 December 2021 Accepted: 10 January 2022 Publication: 28 February 2022

retreat but not; if knowing difficulties and being prepared, it is still feasible. The second say that "knowing is easy and doing is difficult". In fact, knowing is not difficult, doing is more difficult, and the key is doing.

2.Know and practice the essentials of Taichi

Knowing and practicing Taichi is graceful and easy to learn, which is conducive to popularization; According to the four seasons of spring, summer, autumn and winter, it is divided into four boxing frames. Among the four boxing frames, they are divided into high, medium and low boxing frames according to the way of heaven, earth and people, so as to meet the needs of people of different ages and physical strength; In terms of movement technology, knowing and practicing Taichi is based on the seventy-five style Zhaobao and Style Taichi, which has deleted the complicated and difficult movements, and carefully selected eight styles, namely, preparatory style, King Kong three pairs, lazy tie clothes, right white crane bright wings, single whip, cloud hand, boy worship Guanyin, ending, etc.

The action essentials to be followed in the above eight movements can be summarized as follows: virtual collar top strength, chest and back pulling, shoulder sinking and elbow falling, waist falling and crotch falling, knee loosening and hanging gear, abdomen closing, etc. The lower collar included in the top strength of the virtual collar is to make the chest muscles tense without the upward pulling force, so as to ensure the smoothness of the respiratory tract. In this way, the breath can smoothly pass through the throat, chest and even deep into the lower abdomen (self feeling). Chest and back pulling is also to relax the chest muscles. It means inward and downward, which can make the breathing smooth and sink into the Dantian. Shoulder sinking and elbow dropping, whether in the process of rest or movement, is to prevent the movement of upper limbs from causing pectoral muscle tension and internal air floating. The positive waist can adjust the physiological radian of the lumbar spine to reduce the stress. The hanging gear can gather the Qi of Dantian. Abdominal contraction can make the lower abdomen tight backward and loose internally, so as to gather internal Qi in the elixir field.

In a word, only through the eight types and six essentials can the practitioners achieve the ideal state of Qi sinking into the Dantian and spiritual unity, and continuously stimulate the enthusiasm of self-exercise and improve the effect of fitness and mental cultivation on the basis of pursuing the perfection of action standards and technology.

3. Characteristics of knowing and doing Taichi

(1) Traditional. Knowing and practicing Taichi, adhering to China's traditional culture, especially draws the essence of Confucianism, Buddhism and Taoism, not only preserves the connotation of traditional Taichi, but also highlights its own unique style of routine technology, and constantly sublimes it. It can also combine knowledge and practice Taichi with traditional calligraphy and painting, which can also achieve the effect of self-cultivation and heart cultivation.

(2) Scientific. Knowing and practicing Taichi routine action arrangement requirements 
Article History: Received: 28 October 2021 Revised: 05 December 2021 Accepted: 10 January 2022 Publication: 28 February 2022

comply with the principles of human physiology, biomechanics and anatomy. The movements are beautiful and coordinated, which is conducive to strengthening physique and improving physical and psychological quality. In addition, knowing and practicing Taichi is in line with the internal requirements of the law of human movement. The routine moves are from simple to complex, from easy to difficult. For beginners, you can start from the foundation. Eight movements are simple and easy, and you can sweat slightly in 3 minutes; For advanced learners, it can be combined with the four seasons, calligraphy and painting to sublimate their actions. No matter at which stage, they can achieve appropriate and moderate amount of exercise and have the key points of scientific movement.

\section{INVESTIGATION AND RESEARCH}

Through literature research and investigation, this paper summarizes the traditional sports health preservation methods, summarizes the characteristics of various schools of traditional Taichi and their respective characteristics of physical and mental health care, compares the similarities and differences between traditional sports health preservation and modern sports health preservation methods, and analyzes the similarities, differences and advantages. The knowledge and practice Taichi group, modern exercise group and blank control group were established for six months and no less than 210 minutes a week; The blank control group did not exercise regularly. Before and after the investigation, SCL-90 symptom checklist was used to evaluate the physical and mental changes of each group before and after the experiment.

This paper discusses the role of Taichi in cultivating body and mind from the perspective of modern psychology such as balancing consciousness and subconsciousness. The localization of western psychology, the use of a set of knowledge and practice Taichi, which integrates traditional Chinese medicine health culture and Western medicine cognitive behavior therapy, the combination of body and function, and the co cultivation of mind and body, has been developed into a localized cognitive behavior therapy. Combined with the methods of modern psychology, the comparative study of Chinese and western sports health preservation methods has been carried out.

Among the 143 people investigated, there were 53 in the knowledge and practice Taichi group, including 24 males, accounting for $45.3 \%$ and 29 females, accounting for 54.7\%, with an average age of $46.85 \pm 10.929$ years; There were 5fi people in the modern sports group, including 29 men, accounting for $51.8 \%$, and 27 women, accounting for $48.2 \%$. The average age was $44.86 \pm 8.45$ years old; There were 34 people in the blank control group, 21 men, accounting for fil. $8 \%$, and 13 women, accounting for $38.2 \%$. The average age was $41.68 \pm$ 9.897 years old.

\section{TABLE I. Basic information of gender and age of the three groups of subjects}


Article History: Received: 28 October 2021 Revised: 05 December 2021 Accepted: 10 January 2022 Publication: 28 February 2022

\begin{tabular}{|c|c|c|c|c|}
\hline \multirow[b]{2}{*}{ GROUPING } & \multicolumn{2}{|c|}{ GENDER } & \multirow[b]{2}{*}{ AGE } & \multirow{2}{*}{$\begin{array}{c}\text { TOTAL } \\
\text { NUMBER }\end{array}$} \\
\hline & $\begin{array}{l}\text { Male } \\
(\%)\end{array}$ & $\begin{array}{c}\text { Female } \\
(\%)\end{array}$ & & \\
\hline $\begin{array}{l}\text { ZHIXING Taichi } \\
\text { GROUP }\end{array}$ & $24(45.3)$ & $29(54.7)$ & $46.85 \pm 10.929$ & 53 \\
\hline $\begin{array}{c}\text { MODERN SPORTS } \\
\text { GROUP }\end{array}$ & $29(51.8)$ & 2748.2) & $44.86 \pm 8.645$ & 56 \\
\hline $\begin{array}{c}\text { BLANK CONTROL } \\
\text { GROUP }\end{array}$ & $21(61.8)$ & $13(38.2)$ & $41.68 \pm 9.897$ & 34 \\
\hline
\end{tabular}

Therefore, there is no significant difference in gender, age and other basic information among the knowledge and practice Taichi group, modern exercise group and blank control group.

The data of SCL-90 symptom checklist filled in before the experiment were buried and counted. It was known that the total symptom score of Taichi group was $154.79 \pm 64.392$; The total score of modern sports group is $137.46 \pm 60.781$; The total score of the blank control group was $122.18 \pm 34.633$ (see Table 2). From the numerical point of view, before the test, the degree of physical and mental health problems in the three groups was: knowledge and practice Taichi group > modern exercise group > blank control group. Through the analysis of variance, $\mathrm{f}=3.474, \mathrm{P}=0.034<0.05$, which was statistically significant. $\mathrm{q}$ test for pairwise comparison of the mean values of multiple samples. According to the level of $\mathrm{a}=0.05$, it can be considered that the mean values of the knowledge and practice Taichi group and the modern exercise group are higher than those of the blank control group, but it can not be considered that the mean values are different between the blank control group and the modern exercise group, and between the modern exercise group and the knowledge and practice Taichi group (see Table 3 ). That is, before the investigation and test, the symptom degree of the respondents in the knowledge and practice Taichi group and modern exercise group was higher than that in the blank control group.

TABLE II. The total score of symptoms of the three groups before and after the experiment

\begin{tabular}{|c|c|c|}
\hline GROUPING & $\begin{array}{c}\text { TOTAL SCORE OF } \\
\text { SYMPTOMS BEFORE } \\
\text { EXPERIMENT }\end{array}$ & $\begin{array}{c}\text { TOTAL SCORE OF } \\
\text { SYMPTOMS AFTER } \\
\text { EXPERIMENT }\end{array}$ \\
\hline $\begin{array}{c}\text { ZHIXING Taichi } \\
\text { GROUP }\end{array}$ & $154.79 \pm 64.392$ & $118.51 \pm 25.362$ \\
\hline $\begin{array}{c}\text { MODERN SPORTS } \\
\text { GROUP }\end{array}$ & $137.46 \pm 60.781$ & $108.52 \pm 19.250$ \\
\hline
\end{tabular}


Article History: Received: 28 October 2021 Revised: 05 December 2021 Accepted: 10 January 2022 Publication: 28 February 2022

BLANK CONTROL GROUP

TABLE III. Q-test for comparing the mean of total symptom scores of the three groups before the experiment

\begin{tabular}{|c|c|c|c|}
\hline Grouping & $\mathbf{N}$ & $\mathbf{1}$ & $\mathbf{2}$ \\
\hline $\begin{array}{c}\text { ZHIXING Taichi } \\
\text { GROUP }\end{array}$ & 34 & 122.18 & 137.46 \\
\hline $\begin{array}{c}\text { MODERN SPORTS } \\
\text { GROUP }\end{array}$ & 56 & 137.46 & 154.79 \\
\hline $\begin{array}{c}\text { BLANK CONTROL } \\
\text { GROUP }\end{array}$ & 53 & 0.205 & 0.151 \\
\hline SIG. & & & \\
\hline
\end{tabular}

The data of SCL-90 symptom checklist filled in after the experiment were sorted and counted. It was known that the total symptom score of Taichi group was 118,51 \pm 25.362 ; The total score of modern sports group is $108.52 \pm 19.250$; The total score of the blank control group was $119.82 \pm 33.255$, and there was a downward trend in the three groups. By analysis of variance, $f=2.942, P=0.056>0.05$, there was no statistical significance. There was no difference in the total symptom score between the groups after the experiment. From the integral point of view, the degree of symptoms of the subjects after the experiment were: blank control group > Zhixing Taichi group > modern sports group, and the structure changed before the experiment.

\section{DISCUSSION AND PROSPECT}

From the change of each symptom factor, knowing and practicing Taichi has the best effect on improving physical symptoms, and also has obvious improvement effect on interpersonal sensitivity symptoms, depression symptoms, anxiety symptoms and paranoid symptoms. From the change of integral, it is better than the modern exercise group. In addition, Zhixing Taichi group also had a good effect on diet and sleep. The reasons for this result mainly include the following aspects:

First, environmental factors. The practice field of Zhixing Taichi is relatively spacious, and outdoor exercises are often held; Modern sports adopt some fitness equipment, mainly indoor, and the emission of equipment is relatively tight, which affects the air circulation. It can be said that good air circulation is the catalyst to promote good health preservation effect. Some scholars have also shown that when people are in a green environment, that is, where there are 
Article History: Received: 28 October 2021 Revised: 05 December 2021 Accepted: 10 January 2022 Publication: 28 February 2022

high negative ions in the air, the recovery speed of physical fatigue will also be accelerated.

Second, both form and spirit. Knowing and practicing Taichi not only pays attention to the activities of physical movement, but also pays more attention to the unity of meaning, Qi and form, that is, it also cooperates with the cultivation of God while physical activities; Most modern sports are physical exercises based on fitness. Health is not only the absence of physical diseases, but also mental health, social adaptability, morality and other mental and psychological factors. Form cultivation is the basic stage of health preservation, while spirit cultivation is the advanced stage of health preservation. Only by cultivating both form and spirit can we achieve the "end of life" as stated in the internal classic.

At present, China has entered the ranks of aging countries. In view of the huge population base, the number of elderly population in China will be very considerable, its health status is worrying, and the disability rate and disability rate will also increase sharply. Under the fastpaced and high-pressure urban life, with the increasing number of elderly chronic diseases and sub-health, social medical insurance expenditure is huge, medical expenses are rising, individuals, collectives, society and the government are overwhelmed, and it has become the norm that it is difficult and expensive to see a doctor. However, the existing medical and health service system can not meet the health needs of contemporary people. It is urgent to upgrade the old medical system - health management came into being.

The service content of health management shall cover physical examination, health lectures, health assessment, health pre-treatment, chronic disease management, sub-health conditioning, family doctors, etc. Through the establishment of health service market, the growth of professional and technical talents, the effective integration of market demand and service resources, and the joint assistance of insurance industry, information industry, network technology and health management industry. It will effectively promote the marketization process of health management industry and establish a health management operation mode and service system with Chinese characteristics and in line with national needs.

\section{CONCLUSION}

The research on ancient and modern literature shows that the exercise method of emphasizing the correspondence between heaven and man, the combination of movement and static, and the joint cultivation of form and spirit is the real exercise and health preservation method in line with the concept of health preservation. Knowing and practicing Taichi is the inheritance and innovation of traditional Taichi, and inherits the eight advantages of Taichi, which means "lightness, flexibility, justice and peace". From creation to development, they respect and follow the supreme health preservation principle of unity of body and mind and unity of knowledge and practice, which is more in line with the way of traditional Chinese medicine. Its effect of regulating body and mind is better than some modern sports and fitness 
Article History: Received: 28 October 2021 Revised: 05 December 2021 Accepted: 10 January 2022 Publication: 28 February 2022

methods that only emphasize fitness and strengthen the body. It has important guiding significance for modern people's sports and health preservation in cognition and practice.

\section{REFERENCES}

[1] Chacon, R., Codony, D., \& Toledo, A., (2017). From physical to digital in structural engineering classrooms using digital fabrication. Computer Applications in Engineering Education, 25(6), 927937.

[2] Ahmed, \& Ali, A., (2015). A comparative study of qos performance for location based and corona based real-time routing protocol in mobile wireless sensor networks. Wireless Networks, 21(3), 1015-1031.

[3] Friedman, A., Hahn, K. A., Etz, R., Rehwinkel-Morfe, A. M., Miller, W. L., \& Nutting, P. A., et al. (2014). A typology of primary care workforce innovations in the united states since 2000. Medical Care, 52(2), 101.

[4] Wong, L. H., Chai, C. S., Zhang, X., \& King, R. B., (2015). Employing the tpack framework for researcher-teacher co-design of a mobile-assisted seamless language learning environment. IEEE Transactions on Learning Technologies, 8(1), 31-4

[5] Ding, Y., Li, Y., \& Cheng, L., (2020). Application of internet of things and virtual reality technology in college physical education. IEEE Access, PP(99), 1-1.

[6] Kim, H., Shin, H., Kim, H. S., \& Kim, W. T., (2018). Vr-cpes: a novel cyber-physical education systems for interactive vr services based on a mobile platform. Mobile Information Systems, 2018(PT.3), 1-10.

[7] Ji, X., (2019). Community guidance model based on interactive multimedia system. Multimedia Tools and Applications, 78(4), 4723-4741.

[8] Chang, C. K., Reisman, S., \& Tovar, E., (2017). Advances in learning technologies. Computer, 50(5), 14-17. 